\title{
Silence is Golden: Branching Bisimilarity is Decidable for Context-Free Processes
}

\author{
Hans Hüttel * \\ Laboratory for the Foundations of Computer Science, JCMB \\ University of Edinburgh, Edinburgh EH9 3JZ \\ SCOTLAND
}

\begin{abstract}
We show that the branching bisimulation equivalence introduced by Rob van Glabbeek is decidable for the class of normed, recursively defined BPA processes with silent actions, thus generalizing the decidability result for strong bisimilarity by Baeten, Bergstra, and Klop.
\end{abstract}

\section{Introduction}

In their paper [BBK87] Baeten, Bergstra, and Klop showed that strong bisimulation equivalence is decidable for normed recursively defined BPA processes [BK88], a class of processes corresponding to that of irredundant context-free grammars without empty productions. In this paper we generalize this result by showing that the branching bisimilation equivalence introduced by van Glabbeek and Weijland in [vGW89] is decidable for the class of normed recursively defined BPA processes with silent actions. The proof generalizes that of the decidability of strong bisimulation equivalence given by Colin Stirling and the present author in [HS91], relying as it does on a similar tableau-based decision method. This tableau method is related to the equivalence algorithms for certain classes of context-free grammars introduced by Korenjak and Hopcroft [KH66], and it directly reflects usual intuitions about determining the equivalence of processes by means of successive matchings of transitions.

Since the class of processes considered allows infinite state spaces, the present result further underpins the fact that the decidability of behavioural equivalences can extend beyond the finite-state case, and that decidability should be seen as a criterion for determining the relative merits and deficiencies of behavioural equivalences.

Section 2 introduces the notion of branching bisimilarity and the class of normed BPA rec processes. In Section 3 we describe the tableau system, prove its soundness and completeness, give a complexity measure and establish the decidability result of branching bisimilarity. Section 4 sums up conclusions and gives suggestions for further work.

\footnotetext{
*Present address: Department of Mathematics and Computer Science, Aalborg University Centre, Fredrik Bajersvej 7E, 9220 Aalborg $\emptyset$, Denmark. E-mail: hansoiesd.auc.dK
} 


\section{Preliminaries}

\subsection{Branching bisimilarity}

The processes that we will be looking at have their behavioural semantics given by transition graphs with silent actions. For comparison we first describe the notion of weak bisimulation equivalence, introduced by Milner [Mil80, Mil89], which is essentially bisimulation equivalence defined on the derived weak transition relations that disregard silent actions.

Definition 2.1 For a transition graph $\mathcal{G}=(\operatorname{Pr}, A c t \cup\{\tau\}, \rightarrow)$ with silent action $\tau$, the weak transition relations $\{\stackrel{a}{\Rightarrow} \mid s \in$ Act $\cup\{\epsilon\}\}$ are given by $\stackrel{a}{\longrightarrow}=\stackrel{\tau}{\rightarrow} \stackrel{a}{\rightarrow} \stackrel{\sim}{\rightarrow}$ for $a \in$ Act and $\stackrel{c}{\Longrightarrow}=\stackrel{\sim}{\rightarrow}$

In the definition below, we use the 'observational' mapping $\phi:(\text { Act } \cup\{\tau\})^{*} \rightarrow$ Act which is the homomorphic extension of the function defined by $\phi(a)=a$ for $a \in A c t$ and $\phi(\tau)=\epsilon$.

Definition 2.2 [Mil89] $A$ weak bisimulation on $\mathcal{G}$ is a symmetric relation $R \subseteq \operatorname{Pr} \times \operatorname{Pr}$ such that whenever $p R q$ for any $a \in A c t \cup\{\tau\}$ we have that $p \stackrel{a}{\rightarrow} p^{\prime}$ implies that there exists a $q^{\prime}$ such that $q \stackrel{\phi(a)}{\Longrightarrow} q^{\prime}$ with $p^{\prime} R q^{\prime}$. We define $\approx b y$

$$
\approx=\{(p, q) \mid p R q \text { for some weak bisimulation } R\}
$$

If $p \approx q$ we say that $p$ and $q$ are weakly bisimilar.

The notion of branching bisimilarity was put forward by van Glabbeek and Weijland in [vGW89] as an alternative to weak bisimulation.

Definition 2.3 [vGW89] $A$ branching bisimulation (bb) on $\mathcal{G}$ is a symmetric relation $R \subseteq \operatorname{Pr} \times \operatorname{Pr}$ such that whenever $p R q$ for any $a \in A$ ct $\cup\{\tau\}$ we have that $p \stackrel{a}{\rightarrow} p^{\prime}$ implies

- $a=\tau$ and $p^{\prime} R q$ or

- there exist $q_{1}^{\prime}, q^{\prime}$ such that $q \stackrel{\varrho}{\Rightarrow} q_{1}^{\prime} \stackrel{a}{\rightarrow} q^{\prime}$ with $p R q_{1}^{\prime}, p^{\prime} R q^{\prime}$

We define $\approx_{b}$ by

$$
\approx_{b}=\{(p, q) \mid p R q \text { for some } b b R\}
$$

If $p \approx_{b} q$ we say that $p$ and $q$ are branching bisimilar.

Unlike weak bisimulation equivalence, changes in branching properties caused by individual $\tau$ transitions must always be taken into account in branching bisimulation. (Example 2.2 provides an example of the importance of this, namely two processes that are weakly bisimilar but not branching bisimilar). An equivalent definition which reflects this stuttering property better is the one below which we will be using in the tableau system presented in Section 3.

Proposition $2.1 A$ branching bisimulation on $\mathcal{G}$ is a symmetric relation $R \subseteq \operatorname{Pr} \times \operatorname{Pr}$ such that whenever $p R q$ for any $a \in A c t \cup\{\tau\}$ we have that if $p \stackrel{a}{\rightarrow} p^{\prime}$ then either

- $a=r$ and $p^{\prime} R q$ or

- there exist $q_{0}^{\prime}, \ldots, q_{n}^{\prime}, q^{\prime}$ such that $q=q_{0}^{\prime} \stackrel{\tau}{\rightarrow} q_{1}^{\prime} \stackrel{\tau}{\rightarrow} \ldots \stackrel{r}{\rightarrow} q_{n}^{\prime} \stackrel{a}{\rightarrow} q^{\prime}$ with $p R q_{i}^{\prime}$ for $0 \leq i \leq n$ and $p^{\prime} R q^{\prime}$. 


\subsection{Normed BPA rec $^{\tau}$}

Recursive Basic Process Algebra (BPA) with silent actions, the class BPA $_{\text {rec }}^{r}$ [BK88], consists of processes given by systems of defining equations $\Delta=\left\{X_{i} \stackrel{\text { def }}{=} E_{i} \mid 1 \leq i \leq m\right\}$. The process expressions $E_{i}$ are given by the syntax

$$
E::=a|\tau| E_{1}+E_{2}\left|E_{1} E_{2}\right| X
$$

where $\tau$ is a new, silent action not in Act. In the following, elements of $V a r^{*}$ will be denoted by Greek letters: $\alpha, \beta, \ldots$ and BPA expressions in general by $E, F \ldots$ The operational semantics given by the transition relations $\{\stackrel{a}{\rightarrow} \mid a \in A c t \cup\{\tau\}\}$ is as given below.

Definition 2.4 Any system of BPA process equations $\Delta$ defines a labelled transition graph. The transition relations are given as the least relations satisfying the following rules:

$$
\begin{array}{cc}
\frac{E \stackrel{a}{\rightarrow} E^{\prime}}{E+F \stackrel{a}{\rightarrow} E^{\prime}} & \frac{F \stackrel{a}{\rightarrow} F^{\prime}}{E+F \stackrel{a}{\rightarrow} F^{\prime}} \\
\frac{E \stackrel{a}{\rightarrow} E^{\prime}}{E F \stackrel{a}{\rightarrow} E^{\prime} F} & a \stackrel{a}{\rightarrow} \epsilon \quad a \in A c t \cup\{\tau\} \\
\frac{E \stackrel{a}{\rightarrow} E^{\prime}}{X \stackrel{a}{\rightarrow} E^{\prime}} & X \stackrel{\text { def }}{=} E \in \Delta
\end{array}
$$

We restrict our attention to weakly normed systems of process equations.

Definition 2.5 The weak norm of any $X \in V$ ar is given by

$$
\|X\|=\min \left\{\text { length }(w) \mid X \stackrel{w}{\Longrightarrow} \epsilon, w \in A c t^{*}\right\}
$$

A system of defining equations $\Delta$ is weakly normed if for any $X \in \operatorname{Var} 0<\|X\|<\infty$. The maximal norm of any variable in $\Delta$ is defined by $M_{\Delta}=\max \{\|X\| \mid X \in V$ ar $\}$.

Since norms must be strictly positive, all variables must eventually perform an observable action and processes can therefore not terminate silently.

In Section 3 we shall also need the notion of a strong norm (cf. [HS91]).

Definition 2.6 The strong norm of a $B P A_{\text {rec }}^{\tau}$ expression $E$ is defined as

$$
|E|=\min \left\{\text { length }(w) \mid E \stackrel{w}{\rightarrow} \epsilon, w \in A c t^{+}\right\}
$$

A system of defining equations $\Delta$ is strongly normed if for any variable $X \in \operatorname{Var}|X|<\infty$. The maximal strong norm of any variable in $\Delta$ is $m_{\Delta}=\max \{|X| \mid X \in V a r\}$.

Clearly, if $\Delta$ is weakly normed it is also strongly normed.

Finally, we restrict our attention to systems of defining equations given in 3-Greibach Normal Form (3-GNF). 
Definition 2.7 A system of BPA equations $\Delta$ is said to be in Greibach Normal Form (GNF) if all equations are of the form

$$
\left\{X_{i} \stackrel{\text { def }}{=} \sum_{j=1}^{n_{i}} a_{i j} \alpha_{i j} \mid 1 \leq i \leq m\right\}
$$

If for each $i, j$ the variable sequence $\alpha_{i j}$ has length $\left(\alpha_{i j}\right)<k, \Delta$ is said to be in $k$-GNF.

The normal form is called Greibach Normal Form by analogy with context-free grammars (without the empty production) in Greibach Normal Form (see e.g. [HU79]). There is an obvious correspondence with grammars in GNF: process variables correspond to non-terminals, the root is the start symbol, actions correspond to terminals, and each equation $X_{i} \stackrel{\text { def }}{=} \sum_{j=1}^{n_{i}} a_{i j} \alpha_{i j}$ can be viewed as the family of productions $\left\{X_{i} \rightarrow a_{i j} \alpha_{i j} \mid 1 \leq j \leq n_{i}\right\}$. The notion of normedness says that the grammar must not have useless productions. The requirement of norms being positive is in analogy with the requirement that a grammar has no empty productions.

It is well-known that any context-free language (without the empty string) is generated by a grammar in 3-GNF [HU79]. One can show that actually is not a real restriction, since any system of process equations $\Delta$ in $\mathrm{BPA}_{\mathrm{rec}}^{\tau}$ can effectively be rewritten to a $\Delta^{\prime}$ which is strongly bisimilar to $\Delta$ and therefore weakly normed iff $\Delta$ is [BBK87]. This leaves us with transition graphs whose states are strings of process variables; the further restriction to variable sequences of length at most 2 guarantees limited growth when determining single transitions:

Proposition 2.2 Suppose $\Delta$ is in 3-GNF. Then, for any $\alpha \in \operatorname{Var}^{*}$, whenever $\alpha \stackrel{\text { a }}{\rightarrow} \alpha^{\prime}$ we have length $\left(\alpha^{\prime}\right) \leq$ length $(\alpha)+1$.

Because weak norms are assumed strictly positive, we have a simple relationship between lengths and norms:

Proposition 2.3 For $\alpha \in V a r^{*}$ length $(\alpha) \leq\|\alpha\|$ and $\|\alpha\| \leq M_{\Delta}$ length $(\alpha)$.

The weak norm is additive under sequential composition:

Proposition 2.4 For $\alpha, \beta \in \operatorname{Var}^{*}\|\alpha \beta\|=\|\alpha\|+\|\beta\|$.

Note that for weakly normed systems we have

Proposition $2.5 \alpha \approx_{b} \beta$ implies that $\|\alpha\|=\|\beta\|$.

Example 2.1 Consider $\Delta_{1}=\{A \stackrel{\text { def }}{=} a+b B C ; B \stackrel{\text { def }}{=} \tau A ; C \stackrel{\text { def }}{=} c\}$ and $\Delta_{2}=\{X \stackrel{\text { def }}{=} a+b X Y ; Y \stackrel{\text { def }}{=}$ c\}. The transition graphs are shown in Figure 1. For $\Delta_{1}$ we have $L_{o b s}(A)=\left\{b^{n} a c^{n} \mid n \geq 0\right\}$, $L_{o b s}(B)=L_{o b s}(A)$ and $L_{o b s}(C)=\{c\} . X \approx_{b} A$ because of the branching bisimulation

$$
\left\{\left(X Y^{n}, A C^{n}\right) \mid n \geq 0\right\} \cup\left\{\left(X Y^{n}, B C^{n}\right) \mid n \geq 1\right\} \cup\left\{\left(Y^{n}, C^{n}\right) \mid n \geq 1\right\} \cup\{(\epsilon, \epsilon)\}
$$

For the tableau system we need the following results - firstly, $\approx_{b}$ is a congruence w.r.t. sequential composition: 


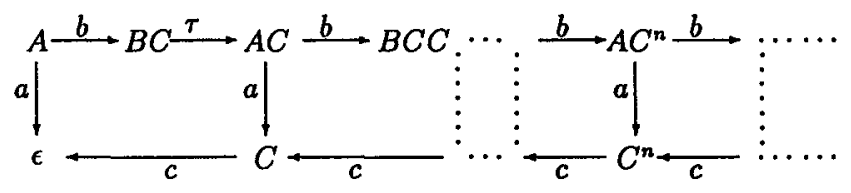

Figure 1: Transition graph for $A \stackrel{\text { def }}{=} a+b B C ; B \stackrel{\text { def }}{=} \tau A ; C \stackrel{\text { def }}{=} c$

Proposition 2.6 If $\alpha_{1} \approx_{b} \beta_{1}$ and $\alpha_{2} \approx_{b} \beta_{2}$ then $\alpha_{1} \alpha_{2} \approx_{b} \beta_{1} \beta_{2}$

The other result is a 'split' lemma that allows us to discard identical tails:

Lemma 2.1 If $\alpha_{1} \alpha \approx_{b} \alpha_{2} \alpha$ then $\alpha_{1} \approx_{b} \alpha_{2}$.

It is important to note that this does not hold for weak bisimulation. The following counterexample arose in a discussion with Kim Larsen and is due to him.

Example 2.2 Consider $\Delta=\{X=a Y, Y=a+\tau X, A=a+a B, B=a\}$. As $\|X\|=2,\|Y\|=$ $1,\|A\|=1$ and $\|B\|=1, \Delta$ clearly obeys all requirements stated above. It is easily seen that $X \approx$ $B Y$ and that $A \not z B$. However, we have $A Y \approx B Y$, since $\{(A Y, B Y),(B Y, X),(Y, Y),(\epsilon, \epsilon),(X, X)\}$ is a weak bisimulation. The problem lies in the fact that weak bisimilarity does not require the results of intermediate steps in weak transitions to be related. In particular, $A Y \stackrel{a}{\rightarrow} Y$ is matched by $B Y \stackrel{a}{\Rightarrow} X$. The latter is due to $B Y \stackrel{a}{\rightarrow} Y \stackrel{\tau}{\rightarrow} X$, where we clearly have that $A Y \not \approx$.

\section{A tableau system for branching bisimulation}

\subsection{Building tableaux}

A tableau for determining branching bisimilarity is a maximal proof tree built using the proof rules in Table 1. Tableaux consist of a number of subtableaux. These are built from successive applications of the STEP rule.

STEP is applicable iff there is a possibility of matching transitions. A possible match is any set of equations whose sides are the results of successful matching transitions according to the definition of branching bisimilarity in Proposition 2.1:

Definition 3.1 A set of equations $M$ is a possible match for $\alpha=\beta$ if for any a $\in$ Act we have that if $\alpha \stackrel{a}{\rightarrow} \alpha^{\prime}$ then either

- $a=\tau$ and $\alpha^{\prime}=\beta \in M$ or

- there exist $\beta_{0}^{\prime}=\beta, \ldots, \beta_{n}^{\prime}, \beta^{\prime}$ such that $\beta_{0} \stackrel{\tau}{\rightarrow} \beta_{1}^{\prime} \stackrel{\tau}{\rightarrow} \cdots \stackrel{\tau}{\rightarrow} \beta_{n}^{\prime} \stackrel{a}{\rightarrow} \beta^{\prime}$ with $\alpha=\beta_{i}^{\prime} \in M$ for $0 \leq i \leq n$ and $\alpha^{\prime}=\beta^{\prime} \in M$.

and similarly for any $\beta \stackrel{a}{\rightarrow} \beta^{\prime}$. 
This definition appears to allow infinitely many possible matches, since there seems to be no bound on the length $n$ of a matching transition sequence. However, this is not the case. Firstly, we have

Proposition 3.1 If $\alpha \approx_{b} \beta$ we can find a possible match $M$ for $\alpha=\beta$ such that whenever $\alpha \stackrel{a}{\rightarrow} \alpha^{\prime}$ is matched by $\beta_{0}^{\prime}=\beta, \ldots, \beta_{n}^{\prime}, \beta^{\prime}$ such that $\beta_{0} \stackrel{\tau}{\rightarrow} \beta_{1}^{\prime} \stackrel{\tau}{\rightarrow} \ldots \stackrel{\tau}{\rightarrow} \beta_{n}^{\prime} \stackrel{a}{\rightarrow} \beta^{\prime}$ with $\alpha=\beta_{i}^{\prime} \in M$ for $0 \leq i \leq n$ and $\alpha^{\prime}=\beta^{\prime} \in M$ all $\beta_{i}^{\prime}(0 \leq i \leq n)$ are distinct.

Secondly, we have

Proposition 3.2 If $X \alpha \approx_{b} Y \beta$ and $X \alpha \stackrel{a}{\rightarrow} \alpha^{\prime} \alpha$ is matched by $Y \beta \stackrel{c}{\Rightarrow} \beta_{1}^{\prime} \beta \stackrel{a}{\rightarrow} \beta^{\prime} \beta$ any intermediate state $\beta^{\prime \prime}$ in $Y \beta \Longrightarrow \beta_{1}^{\prime} \beta$ has length $\left(\beta^{\prime \prime}\right) \leq M_{\Delta}+$ length $(\beta)$. Furthermore, length $\left(\beta^{\prime}\right) \leq M_{\Delta}+$ length $(\beta)+1$

The outbranching is a multiple of the bound $B_{X, Y}$ on the number of single transition steps for $X \alpha=Y \beta$; this factor only depends on the leftmost variables and is given by

$$
B_{X, Y}=\left\{\alpha^{\prime} \mid X \stackrel{a}{\rightarrow} \alpha^{\prime}, a \in A c t \cup\{\tau\}\right\} \cup\left\{\beta^{\prime} \mid Y \beta \stackrel{a}{\rightarrow} \beta^{\prime}, a \in \operatorname{Act} \cup\{\tau\}\right\}
$$

Proposition 3.3 Let $v$ be the cardinality of Var. If $X \alpha \approx_{b} Y \beta$, there is a possible match for $X \alpha=$ $Y \beta$ with at most $B_{X, Y} \sum_{j=2}^{2 K}(j-1) v^{j}$ equations, where $K=M_{\Delta}+1+\max ($ length $(\alpha)$, length $(\beta))$.

Clearly, STEP is forwards sound in the following sense:

Proposition 3.4 (Forwards soundness of STEP) If $\alpha \approx_{b} \beta$, then there is a possible match $M$ such that whenever $\alpha^{\prime}=\beta^{\prime} \in M$ we have $\alpha^{\prime} \approx_{b} \beta^{\prime}$.

An eliminating subtableau for $X \alpha=Y \beta$ consists of attempted matches to the depth where an equation of the form $\alpha=\gamma \beta$ is reached. When $|X| \leq|Y|$ each non-residual leaf of an eliminating subtableau for $X \alpha=Y \beta$ is either labelled $\alpha=\gamma \beta$ (a residual of the subtableau), or $\alpha_{i} \alpha=\beta_{i} \beta$. Because the number of successive attempted matches is $|X|$ there is at least one residual and since all norms are strictly positive, $\alpha$ and $\beta$ must persist as suffixes throughout the subtableau. For any such subtableau we pick one residual node and call it the residual. If instead $|Y|<|X|$ the same holds, only now the residual is $\gamma \alpha=\beta$. Unless a subtableau leaf is a successful terminal (Definition 3.3 below) it is used as the basis of a new subtableau. However, before a new subtableau is constructed, for every leaf one of the SUB rules is used to trim the length of the expressions in the new subtableau root. From Propositions 2.6 and 2.1 we see that the SUB rules are forwards sound in the following sense:

Proposition 3.5 (Soundness of SUBL and SUBR) If $\alpha_{i} \alpha \approx_{b} \beta_{i} \beta$ and $\alpha \approx_{b} \gamma \beta$ then $\alpha_{i} \gamma \approx_{b} \beta_{i}$. If $\gamma \alpha_{i} \approx_{b} \beta_{i}$ then $\alpha_{i} \approx_{b} \beta_{i} \gamma$

The rules are only applied to nodes that are not terminal. Terminal nodes can either be successful or unsuccessful.

Definition 3.2 A tableau node is an unsuccessful terminal if it has one of the forms

1. $\alpha=\beta$ with $\|\alpha\| \neq\|\beta\|$

2. $\alpha=\beta$ with $\alpha \neq \epsilon, \beta \neq \epsilon$ and no possible match exists (i.e. STEP is inapplicable). 
Rule within a subtableau

STEP $\quad \frac{\alpha=\beta}{\alpha_{1}=\beta_{1} \ldots \alpha_{k}=\beta_{k}} \quad$ where $\left\{\alpha_{1}=\beta_{1} \ldots \alpha_{k}=\beta_{k}\right\}$

is a possible match for $\alpha=\beta$

Rules for new subtableaux

SUBL $\quad \frac{\alpha_{i} \alpha=\beta_{i} \beta}{\alpha_{i} \gamma=\beta_{i}} \quad$ where $\alpha=\gamma \beta$ is the residual

SUBR $\frac{\alpha_{i} \alpha=\beta_{i} \beta}{\alpha_{i}=\beta_{i} \gamma} \quad$ where $\gamma \alpha=\beta$ is the residual

Table 1: The tableau rules

In both of these cases it is obvious that the expressions compared are not branching bisimilar. Thus, whenever we see an unsuccessful terminal the whole tableau construction aborts.

The nodes that can be successful terminals are those that are potential roots of eliminating subtableaux:

Definition 3.3 $A$ residual or consequent of an application of a SUB rule is a successful terminal if it has one of the forms

1. $\alpha=\beta$ where there is another subtableau root above it on the path from the root also labelled $\alpha=\beta$

2. $\alpha=\alpha$

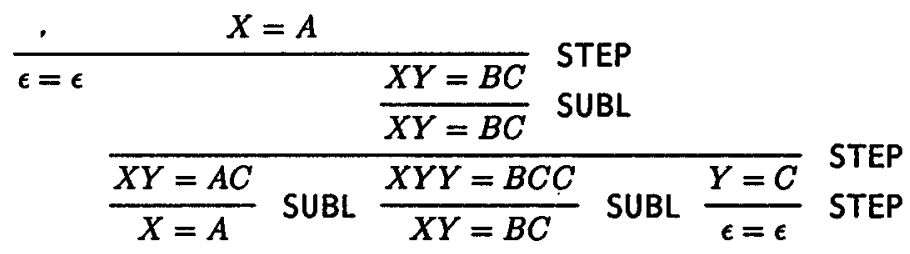

Figure 2: A successful tableau for $X=A$

Example 3.1 (Example 2.1 cont.) The tableau in Figure 2 is a successful tableau for $X=A$. 


\subsection{Termination, completeness, and soundness}

It is important for our decidability result that all tableaux are finite. Since our tableaux are finitely branching by Proposition 3.3, by König's Lemma an infinite tableau would have an infinite path. This would then be caused by the combined absence of unsuccessful termination and the successful termination condition 1 along that path. Since we have assumed 3-GNF and normedness, there is a uniform bound on the total length of the consequent of a SUB rule. Assume wlog that we have a subtableau with root $X \alpha=Y \beta$ and that a SUBL rule was applied to a subtableau leaf:

$$
\frac{\alpha_{1} \alpha=\beta_{1} \beta}{\alpha_{1} \gamma=\beta_{1}} \quad \text { SUBL }
$$

Because the depth of the subtableau is at most $m_{\Delta}$, repeated applications of Proposition 3.2 tell us that length $\left(\alpha_{1}\right) \leq m_{\Delta}\left(M_{\Delta}+1\right)$, length $\left(\beta_{1}\right) \leq m_{\Delta}\left(M_{\Delta}+1\right)$ and length $(\gamma) \leq m_{\Delta}\left(M_{\Delta}+1\right)$. This implies a uniform bound on the length of SUB consequents of $3 m_{\Delta}\left(M_{\Delta}+1\right)$, so there can be no infinite path through infinitely many SUB applications since there are of course only finitely many different equations of any given length. Nor can an infinite path pass through infinitely many residuals. For if a residual $\alpha_{0}=\beta_{0}$ is above the residual $\alpha_{1}=\beta_{1}$ we have that $\left\|\alpha_{0}\right\|=\left\|\beta_{0}\right\|<$ $\left\|\alpha_{1}\right\|=\left\|\beta_{1}\right\|$. By Proposition 2.3, any subsequence of residuals therefore has a uniform bound on the total lengths of expressions compared, again ensuring termination.

Theorem 3.1 For any equation $\alpha=\beta$ all tableaux are finite.

By the forwards soundness of the STEP and SUB rules (Propositions 3.4 and 3.5) we can use the tableau rules in such a way that only valid consequents arise. It is therefore easily seen that the tableau system is complete:

Theorem 3.2 If $\alpha \approx_{b} \beta, \alpha=\beta$ has a successful tableau.

Finally we must show soundness of the tableau system, namely that the existence of a successful tableau for $\alpha=\beta$ indicates that $\alpha \approx_{b} \beta$. This follows from the fact that the tableau system tries to construct a 'bisimulation up to a sequential congruence', which, if a successful tableau is reached, consists of the symmetric closure of the set of nodes in the successful tableau. This notion is the counterpart of the notion of a self-bisimulation used in the tableau system of [HS91] and in [Cau90]. In order to define the corresponding notion for branching bisimulation, we need a simple rephrasing of Proposition 2.1:

Proposition 3.6 A branching bisimulation on a transition graph $\mathcal{G}$ is a symmetric relation $R \subseteq$ $\operatorname{Pr} \times \operatorname{Pr}$ such that whenever $p R q$ for any $a \in A$ ct $\cup\{\tau\}$ we have that if $p=p_{0} \stackrel{\tau}{\rightarrow} p_{1} \stackrel{\tau}{\rightarrow} \cdots p_{m} \stackrel{a}{\rightarrow} p^{\prime}$ then there exist $q_{0}, q_{1}, \ldots, q_{m}, q^{\prime}$ such that $q_{0}=q$ and $p_{i} R q_{i}$ for $1 \leq i \leq m, p^{\prime} R q^{\prime}$ and for $i<m$

$\bullet$

- $q_{i}=q_{i+1}$ or

- there exist $q_{i_{1}}, \ldots, q_{i_{m(i)}}$ such that $q_{i} \stackrel{\tau}{\rightarrow} q_{i_{1}} \stackrel{\tau}{\rightarrow} \cdots q_{i_{m(i)}} \stackrel{\tau}{\rightarrow} q_{i+1}$ with $p_{i} R q_{i j}$ for $1 \leq j \leq n(i)$

and either

- $a=\tau$ and $q_{m}=q^{\prime}$ or 
- there exist $q_{m_{1}}, \ldots, q_{m_{n(m)}}$ such that $q_{m} \stackrel{\tau}{\rightarrow} q_{m_{1}} \stackrel{\tau}{\rightarrow} \cdots q_{m_{m(m)}} \stackrel{a}{\rightarrow} q^{\prime}$

Definition 3.4 For any binary relation $R$ on $V a r^{*}, \vec{R}$ is the least precongruence w.r.t. sequential composition that contains $R, \underset{R}{\longrightarrow}$ the symmetric congruence of $\vec{R}$ and $\overleftrightarrow{R}^{*}$ the transitive closure of $\underset{R}{\longrightarrow}$ and thus the least congruence w.r.t. sequential composition containing $R$.

Definition 3.5 $A$ branching bisimulation up to sequential congruence (sbb) is a symmetric relation $R \subseteq \operatorname{Var} \times \operatorname{Var}$ such that whenever $\alpha R \beta \alpha=\epsilon$ iff $\beta=\epsilon$ and for any $a \in$ Act $\cup\{\tau\}$ we have that if $\alpha=\alpha_{0} \stackrel{\tau}{\rightarrow} \alpha_{1} \stackrel{\tau}{\rightarrow} \cdots \alpha_{m} \stackrel{a}{\rightarrow} \alpha^{\prime}$ then there exist $\beta_{0}, \beta_{1}, \ldots, \beta_{m}, \beta^{\prime}$ such that $\beta_{0}=\beta$ and $\alpha_{i} \underset{R}{\rightarrow} \beta_{i}$ for $1 \leq i \leq m, \alpha^{\prime} \underset{\boldsymbol{R}}{\longrightarrow} \beta^{\prime}$ and for $i<m$

- $-\beta_{i}=\beta_{i+1}$ or

- there exist $\beta_{i_{1}}, \ldots, \beta_{i_{n(i)}}$ s.t. $\beta_{i} \stackrel{\tau}{\rightarrow} \beta_{i_{1}} \stackrel{r}{\rightarrow} \cdots \beta_{i_{n(i)}} \stackrel{\tau}{\rightarrow} \beta_{i+1}$ with $\alpha_{i} \underset{R}{\rightarrow} \beta_{i j}$ for $1 \leq j \leq n(i)$

and either

- $a=\tau$ and $\beta_{m}=\beta^{\prime}$ or

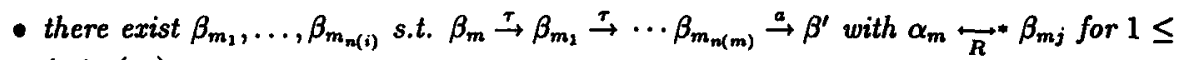
$j \leq n(m)$.

The reason why a bisimulation up to sequential congruence can be said to be an essential part of a bisimulation lies in the following result.

Lemma 3.1 If $R$ is an sbb then $\vec{R}^{*}$ is a bb.

Corollary $3.1 \alpha \approx_{b} \beta$ iff there is an sbb $R$ such that $\alpha R \beta$.

We then have

Theorem 3.3 If $\alpha=\beta$ has a successful tableau $\mathbf{T}$ then

$$
R_{\mathbf{T}}=\left\{\left(\alpha^{\prime}, \beta^{\prime}\right) \mid \alpha^{\prime}=\beta^{\prime} \text { or } \beta^{\prime}=\alpha^{\prime} \text { is an equation in } \mathbf{T}\right\}
$$

is an $s b b$.

So we now get the soundness of the tableau system as

Corollary 3.2 If $\alpha=\beta$ has a successful tableau then $\alpha \approx_{b} \beta$. 


\subsection{Complexity of the tableau system and decidability}

The complexity of the tableau system can be measured in terms of the maximal depth of a tableau, i.e. the length w.r.t. STEP applications of the longest possible path in a successful tableau for an equation $X \alpha=Y \beta$. Let $v$ be the cardinality of $V a r$. By the discussion preceding Theorem 3.1 we have that any SUB consequent has a length of at most $3 m_{\Delta}\left(M_{\Delta}+1\right)$, so an upper bound on the number of distinct SUB consequents along any tableau path is $\sum_{j=2}^{3 m_{\Delta}\left(M_{\Delta}+1\right)}(j-1) v^{j}$. Between any two SUB consequents there can be at most $\left\lceil\frac{3 m_{\Delta}\left(M_{\Delta}+1\right)}{2}\right\rceil$ residuals, so any path that contains SUB consequents can have at most $\left\lceil\frac{3 m_{\Delta}\left(M_{\Delta}+1\right)}{2}\right] \sum_{j=2}^{3 m_{\Delta} M_{\Delta}^{2}+3}(j-1) v^{j}$ subtableau roots. As for the leftmost path, all of whose subtableau roots are residuals, there can be at most max $(\|\alpha\|,\|\beta\|)$ residuals, since the norm of the residuals is strictly decreasing. So, since a subtableau can have a depth w.r.t. STEP applications of at most $m_{\Delta}$, any path can have a length of at most

$$
m_{\Delta} \max \left(\|\alpha\|,\|\beta\|,\left\{\frac{3 m_{\Delta}\left(M_{\Delta}+1\right)}{2}\right\rceil \sum_{j=2}^{3 m_{\Delta}\left(M_{\Delta}+1\right)}(j-1) v^{j}\right)
$$

STEPs.

We also have an upper bound on the outbranching of any tableau for $X \alpha=Y \beta$. This follows from the fact that there is a uniform upper bound on the total length of any subtableau root in any tableau for $X \alpha=Y \beta$. The length of any subtableau root is bounded by

$$
L=\max \left(2 \max (\|\alpha\|,\|\beta\|), 3 m_{\Delta}\left(M_{\Delta}+1\right)\right)
$$

By repeated applications of Proposition 3.2 we see that any node in a subtableau has a length of at most $2 m_{\Delta}\left(M_{\Delta}+1\right)+L$. By Proposition 3.3 this means that there is a uniform upper bound on the number of STEP consequents at any point in any tableau for $X \alpha=Y \beta$ of

$$
\max \left\{B_{X, Y} \mid X, Y \in \operatorname{Var}\right\} \sum_{j=2}^{2 m_{\Delta}\left(M_{\Delta}+1\right)+L}(j-1) v^{j}
$$

This means that any $X \alpha=Y \beta$ has finitely many possible tableaux, so we get the main result

Theorem 3.4 For any weakly normed $\Delta$ it is decidable whether or not $\alpha \approx_{b} \beta$ for $\alpha, \beta \in V a r^{*}$.

The naive decision procedure for $\approx_{b}$ constructs all the finitely many tableaux for $\alpha=\beta$, answering 'yes' if a successful tableau occurs and 'no' otherwise.

\section{Conclusions and directions for further work}

We have here shown that the branching bisimilarity of [vGW89] is decidable for the class of normed BPA processes with silent actions by giving a tableau system. This system has exponential complexity in terms of the longest possible path of a generated tableau; however, in the case of a successful tableau we get additional information in the form of a finite relation whose congruence closure w.r.t. sequential composition is a bisimulation containing the initial equation.

The results of [GH91] show that all known strong equivalences except bisimilarity are undecidable for normed BPA. This means that their weak counterparts also are undecidable, but there are still several open questions for the weak versions of bisimulation equivalence. For branching bisimulation, 
the restriction to processes with strictly positive norms is rather strong, as it rules out the possibility of a process terminating silently. A problem with having nullary norms in the tableau system is that we no longer are guaranteed that $\alpha$ and $\beta$ persist throughout an eliminating subtableau for $X \alpha=Y \beta$, since a match for $X \alpha \stackrel{\text { a }}{\rightarrow}$ may require access to observable actions inside $\beta$. So the natural question is whether there is a way of introducing nullary norms. Moreover, we would of course also want to get rid of the restriction of normedness altogether. However, since this problem also needs to be tackled for strong bisimulation equivalence, it seems that progress must first be made here before we can give any answer for the branching bisimulation case. Last, but not least the questions for weak bisimilarity all remain open. As we saw, Lemma 2.1 does not hold for this equivalence so a different approach must be used in that case.

Finally, it would be interesting if we could give a syntax-directed version of our tableau system for branching bisimulation since this could give us an equational theory of $\approx_{b}$ over normed $\mathrm{BPA}_{\mathrm{rec}}^{r}$ along the lines of [HS91]. A naive approach would be to add the $\tau$-laws for branching bisimulation to the equational theory for strong bisimilarity of [HS91], where a proof system is given that consists of rules that can simulate the tableau construction. However, this theory would not be powerful enough for this; the problem lies in simulating the STEP rule.

\section{References}

[BBK87] J.C.M. Baeten, J.A. Bergstra, and J.W. Klop. Decidability of bisimulation equivalence for processes generating context-free languages. In LNCS 259, pages 93-114. Springer-Verlag, 1987.

[BK88] J.A. Bergstra and J.W. Klop. Process theory based on bisimulation semantics. In J.W. de Bakker, W.P de Roever, and G. Rozenberg, editors, LNCS 354, pages 50-122. SpringerVerlag, 1988.

[Cau90] D. Caucal. Graphes canoniques de graphes algébriques. Informatique théorique et Applications (RAIRO), 24(4):339-352, 1990.

[GH91] J.F. Groote and Hüttel. Undecidable equivalences for basic process algebra. Technical Report ECS-LFCS-91-169, Department of Computer Science, University of Edinburgh, August 1991.

[HS91] H. Hüttel and C. Stirling. Actions speak louder than words: Proving bisimilarity for context-free processes. In Proceedings of 6th Annual Symposium on Logic in Computer Science (LICS 91), pages 376-386. IEEE Computer Society Press, 1991.

[HU79] J. Hopcroft and J.D. Ullman. Introduction to Automata Theory, Languages, and Computation. Addison-Wesley, 1979.

[KH66] A.J. Korenjak and J.E. Hopcroft. Simple deterministic languages. In Proceedings of Seventh Annual IEEE Symposium on Switching and Automata Theory, pages 36-46, 1966.

[Mil80] R. Milner. A Calculus of Communicating Systems, LNCS 92. Springer-Verlag, 1980.

[Mil89] R. Milner. Communication and Concurrency. Prentice-Hall International, 1989.

[vGW89] R.J. van Glabbeek and W.P. Weijland. Branching time and abstraction in bisimulation semantics (extended abstract). In G.X. Ritter, editor, Information Processing 89, pages 613-618. North-Holland, 1989. 